\title{
Philosophiques
}

\section{Genres et tendances}

\section{L'essai : sous-ensemble d'un ensemble}

\section{Roland Houde}

Volume 10, numéro 2, octobre 1983

Le marxisme cent ans après Marx

URI : https://id.erudit.org/iderudit/203236ar

DOI : https://doi.org/10.7202/203236ar

Aller au sommaire du numéro

Éditeur(s)

Société de philosophie du Québec

ISSN

0316-2923 (imprimé)

1492-1391 (numérique)

Découvrir la revue

Citer ce document

Houde, R. (1983). Genres et tendances : l'essai : sous-ensemble d'un ensemble. Philosophiques, 10(2), 403-407. https://doi.org/10.7202/203236ar d'utilisation que vous pouvez consulter en ligne.

https://apropos.erudit.org/fr/usagers/politique-dutilisation/ 
PHILOSOPHIQUES, Vol. X, Numéro 2, Octobre 1983

\title{
INTERVENTION
}

\section{GENRES ET TENDANCES}

\section{L'essai : sous-ensemble d'un ensemble ${ }^{1}$}

\author{
par Roland Houde
}

\begin{abstract}
Nous devons à un philosophe-érudit du $17^{\mathrm{e}}$ siècle une attachante "Lettre " ou "Traité "sur l'Origine des romans, opuscule édité au moins neuf fois entre 1666 ou 1669 et 1699. (Cf. Fabienne Gégou : Lettre-traité de Pierre-Daniel Huet sur l'origine des romans, édition du tricentenaire 1669-1969, Paris, Nizet, 1971). Et il faut bien le constater, depuis Huet (1630-1721),
\end{abstract}

1. Les présupposés historiques, théoriques et méthodiques à la lecture de ce texte écrit pour la S.P.Q. (48 eongrès, ACFAS, U. Laval, 13-16 mai 1980) sont les suivants : (a) l'important essai de notre collègue Louise Marcil-Lacoste, "Hypothèses sur l'historicité du savoir philosophique ", lu à Chicago lors de la réunion annuelle de l'ACPA et maintenant publié dans les Proceedings of the American Catholic Philosophical Association (vol. 52, 1978, p. 20412) dont il nous faut reprendre quelques lignes pour le bénéfice de l'auditeur/lecteur (p. 20910) :

Mon hypothèse ici est la suivante : le savoir philosophique tend à se rapporter au savoir qui se fait en occultant sa propre historicité. La négligence des dictionnaires et encyclopédies philosophiques comme sources à partir desquelles étudier l'histoire de la philosophie correspondrait à un partage des tâches grâce auquel on pourrait occulter ou nier la thérapie par laquelle advient cette historicité. Laissée aux auteurs de dictionnaires et d'encyclopédies, la mise en perspective historique est par ailleurs rejetée comme artificielle, c'est-à-dire synthétique, et sans intérêt, c'est-à-dire périssable.

(b) également important et déterminant sur un autre plan à mon avis son " essai sur l'essai " à paraître dans les Archives des Lettres Canadiennes (Ottawa), travail qu'elle a eu la gentillesse de m'offrir à lire ; (c) une pratique méthodique connue sous le nom d'archilecture ; l'archilecteur étant celui qui s'efforce avec plaisir de constituer le corpus de tout ce qui a été écrit sur une œuvre ou un texte par les lecteurs/critiques. Ce corpus sert alors de matériau sur lequel peut s'exercer une analyse (vérification objective) permettant de cerner tous les éléments de l'œuvre et toutes les tendances (désirs) d'interprétation dans leurs divergences et convergences, d'apercevoir les invariants et les variables, de découvrir le noyau du consensus qui perdure à travers l'exténuation historique (Cf. aussi M. Foucault, Archéologie du savour, 1969, p. 138); (d) en résumé, finalement, mon activité ici sera de réfléchir sur l'énoncé de Bacon prononcé après la parution des Essais de Montaigne (1580) : « Le mot 
les études sur les rapports entre philosophie et littérature ne semblent pas avoir trouvé leur développement nécessaire. Les domaines, petits ou grands, ont leurs clôtures. Pourquoi « ceci est à moi " plutôt que « ceci est à nous "? Une partie de ces études pourrait fort bien aller dans le sens de la distinction entre " genres " et « espèces », entre formes et fonds. Informations et formations des rapports de l'humain au langage, à la science et au monde ${ }^{2}$.

En attendant, de toute façon, il me semble que la préparation d'un texte, quel qu'il soit, dépend d'abord du contrat de lecture/écriture proposé ou décidé ${ }^{3}$. Ceci sera une brève communication sur l'essai. Un essai sur l'essai. Dans l'attente qu'un jour un(e) ou des philosophe(s) d'ici ou d'ailleurs décide(nt) de se pencher sur cette question des "formes " littéraires ou de ces contrats qui indiquent les règles de déchiffrement du texte, les attitudes de lecture, le type d'attente qui sera satisfait, en un mot le "genre " dans lequel le texte s'inscrit (fiction/non

(essai) est nouveau mais la chose est ancienne ". Les mots et les choses toujours. La question se pose, il me semble. Que peut être cette ancienne chose ? Cette ancienne forme? Seraitce le paradoxe? Mon hypothèse donc : Montaigne ne fait que manifester, dans le domaine français, le style, la forme "épidémique " "épigrammatique " ("Measure for Measure", morsure pour morsure) de la Renaissance. De fait, c'est plus qu'une hypothèse. Cf. A.E. Malloch, "The Technique and Function of the Renaissance Paradox", SP, LIII, 1956, p. 191-203 ; Frances A. Yates, Jobn Florio, Cambridge U. Press, 1933 今ou son Giordano Bruno and the Hermetic Tradition, London, Kegan, 1964 ; et Augustus de Morgan, A Budget of Paradoxes (London, 1872 pour la $1^{\text {ère }}$ édition).

2. Ce programme de recherches, parmi d'autres, élargirait sensiblement et positivement l'univers ordonné et clôturé de A.J. Ayer (The Revolution in Pbilosophy, London, MacMillan, 1957, p. 78-8 : "It is science that gives us our knowledge of the world ; there is not, there cannot be, a philosophical brand of knowledge which would compete with science in this field. But where in that case does the philosopher come in ? One thing he can do, of course, is to act as a sort of intellectual policeman, seeing that nobody trespasses into metaphysics". !

3. Le contrat qui gouverne la lecture est plus ou moins explicite. Mais il s'exprime toujours - bien que partiellement par l'apparat de la couverture d'un imprimé : nom d'auteur, titre et sous-titre, titre de collection, nom d'éditeur, lieu, illustrations, préface et tout ce qui figure ou ne figure pas au dos du livre. Exemples: Alexis Mailloux, Essai sur le luxe et la vanité des parures, Québec, Hardy, 1882 ; Albertine Ferland-Angers, Essai sur la poésie religieuse canadienne, Montréal, L'auteur, 1923 ; C.-Roméo Guimont, Droit familial, essai philosophique, Québec, L'Action Sociale, 1921 (2 tomes); Édouard Montpetit, Propos sur la montagne, Essais, Montréal, L’Arbre, 1946 ; Jean Simard, Répertoire, Essai, Montréal, C.L.P., 1961 ; Anne de Nantes, Essai sur le féminisme, préf. du R.P. Y. De La Brière, Avignon, Aubanel, 1926 ; Gilles Deleuze, «Description de la femme », Poésie, no 28, 1945 , p. 28-39. 
fiction, objectif/subjectif, vraisemblable/non vraisemblable). Tout est possible. Mais il me semble peu probable que ce soit le romancier ou le poète qui se charge de cette tâche. Alors pourquoi pas le critique ou philosophe-en-fonction? Conséquemment, je me demande si la philosophie-critique ne s'y retrouverait pas dans son genre et dans sa forme propres ? Essaiparadoxal. Paradoxe du paradoxe. Le désordre se lit dans l'ordre. « Le paradoxe est le pathos ou la passion de la philosophie », rappelle Gilles Deleuze dans Différence et répétition (p. 293). Walter Pater dit que Platon écrivait des essais et que c'était la deuxième manière d'écrire en philosophie après les poèmes plus inspirés de Pythagore, Parménide, Empédocle. Ironie créatrice, méthode de résolution des antinomies. "Il se peut qu'il en soit ainsi », nous dira peut-être un jour Luc Brisson. Sans différence, la répétition est surprenante ! Voici donc la différence que j'essaie ici sur l'essai. L'ambivalence du terme est agréablement parfaite, extrêmement riche. L'essai est à la fois une forme littéraire et une manière de penser. C.Q.F.F. et non pas C.Q.F.D. Car la seule conclusion ou démonstration de l'essai est l'essai lui-même. Le mouvement et le repos du mouvement, de ce mouvement. Sa forme propre : le « paradoxe » ou rencontre des contraires dans un message auto-réflexif. Historiquement constant. En ceci seulement qu'il n'est, qu'il ne vit que s'il se transforme. Et c'est peut-être par et à cause de cette transformation constante qu'historiquement on ne sait que faire de l'essai [tentative, tentation, premier geste ; collectif ; recueil ; titre d'ouvrages, de collection ; expérience à la manière de Robert Lenoble dans son Essai sur la notion d'expérience, Paris, Vrin, 1943 ; traité, fragments, études, mélanges ; le compte rendu, la revue et le " reviewer " ou l'essayeur dans le sens de l'ouvrage de polémique de Galilée (il Saggiatore)] ou plutôt des essayistes qui osent se soumettre à l'insécurité de l'insécurité pour espérer y trouver une jouissance paradoxique. Saisie et dessaisie. Car c'est bien de ça qu'il s'agit. Le cheminement de la pensée. Écriture de pensées ou de fragments (sur tout ou sur rien) ; propositions inouïes, surprenantes, difficiles à croire parce qu'elles choquent les opinions communes.

« Essai », le mot naît avec Montaigne (1580), mais la chose est ancienne remarquera Bacon en 1597. De quelle "chose» 
s'agit-il ? De cette ancienne « forme » littéraire désignée, exposée par Pétrarque, Cicéron, Sénèque, les essais bibliques, les "stoïques"surtout qui ne furent point des «paradoxes" au sens également ancien du nom représentant "une espèce de farceurs » ou "diseurs de bagatelles » ou les " ordinaires " 4 . Dans l'essai, l'extraordinaire peut toujours se démarquer de l'ordinaire. Le contenu est plus grand que le contenant. Le mot long est court. L'ensemble de tous les ensembles n'a pas beaucoup de sens. L'essai/paradoxe, un rapport de transparence entre l'essayiste et le sujet traité ; rapport bref, économique, fragmenté, elliptique, aphoristique, implosif, un tour de phrase à court de traité. Les paradoxes philosophiques réveillent le corps jusque là insensible aux contradictions de son discours, aux contrariétés de la réalité. La même chose est tantôt égale, tantôt inégale. L'assertion généralisée qu'il n'y a pas d'ilôt ou d'arche est elle-même un ilôt ou une arche. La philosophie commence avec le paradoxe et elle s'achève en lui. Le paradoxe est l'épreuve du philosophe. Pascal, 443, éd. Brunschvicg: "Les philosophes, ils étonnent le commun des hommes ; les chrétiens, ils étonnent les philosophes $»^{5}$.

Pour le genre comme pour la forme, pour l'essai comme pour le paradoxe, on ne saurait se contenter de définir ou de répéter à la façon d'un dictionnaire, d'un dictionneur. Il faudrait puiser dans un ensemble donné. De dictionnaires ou d'essais. D'un côté comme de l'autre, le « corpus » est constituable puisque déjà constitué. Constituer un «corpus" au sens de Roland Barthes, c'est se payer une traite. « Ensemble hétéroclite

4. Selon le Dictionnaire universel . . ., de Trévoux, t. 4, p. 520, Paris : 1732.

5. À mon humble avis, il y aurait deux sortes de paradoxes. Ceux qui relèvent d'une recette, qu'en fait on fabrique comme jeux de langage et ceux qui, à y réfléchir, remettent en question ou qui ont une portée. Le fini versus l'infini. Dans ce grand florilège paradoxal du jésuite Marius Bettinus, Apiaria universa philosopbice mathematica, in quibus paradoxa . . ., 1642. Chez Heidegger dans $L ' \hat{E}$ tre et le temps (paradoxe de l'un et de l'autre), p. 150 et 159. Chez Blanchot, "écrire mal, c'est bien écrire " dans "Grève désolée, obscur malaise ", Les Cabiers de la Pléiade, 2, p. 134-7. Chez Baudrillard, À l'ombre des majorités silencieuses ou la fin du social, Paris, Utopie, 1978, p. 36 : "Tel est le sens de leur silence. Mais ce silence est paradoxal - ce n'est pas un silence qui ne parle pas, c'est un silence qui interdit qu'il soit parlé en son nom. Et dans ce sens, loin d'être une forme d'aliénation, c'est une arme absolue ". À comparer avec "L'Infiniment petit mathématique du moyen âge au XIX siècle " et "Les nouveaux infinis mathématiques " dans L'infini et le réel (18 semaine de Synthèse), Paris, Albin Michel, 1955, p. 63-80 et 81-122 respectivement. "Enténébrant la logique/Par la clarté des paradoxes " (Alexis Klimov, Des Arcanes et des jeux, T.-R., Éd. du Bien Public, 1976, p. 147). 
de faits qu'il faudra <traiter> pour en connaître la structure ; cet ensemble doit être défini par le chercheur antérieurement à la recherche : c'est le corpus. Le corpus est une collection finie de matériaux, déterminée à l'avance par l'analyste, selon un certain arbitraire (inévitable) et sur laquelle il va travailler ». (Communications, 4, 1964, p. 133 ou p. 170, Le degré zéro de l'écriture . . ., Éd. Gonthier, 1964). Sans répétition, la différence est également surprenante. L'essai d'aujourd'hui n'est pas celui d'hier. Sans conclusion, je médite l'avant-dernier texte sur l'essai au Québec de 1900-39, sous la signature du littéraire Maurice Lemire (Dictionnaire des cuures . . ., t. II, Montréal, Fides, 1980, p. XXIX) :

C'est surtout en essai que se révèle le véritable sens du régionalisme canadien-français. Si vraiment on avait été d'abord et avant tout attaché aux divers particularismes, on aurait poussé les études ethnographiques tant pour la cueillette que pour l'interprétation. Mais Marius Barbeau fut seul à œuvrer dans ce sens. Les régionalistes furent surtout intéressés par la question nationale. Sauf quelques exceptions, comme l'essai de Louis Lachance, Nationalisme et Religion (1936), il ne s'agit pas d'écrits spéculatifs qui cherchent à élucider une question à partir de théories ou de principes. L'essai, comme les autres genres littéraires, est également un écrit de combat qui a pour but d'aguerrir les troupes et de maintenir leur moral.

Et le dernier texte à cette date, celui de Jean Royer (Le Devoir, 17 mai 1980 , p. 21$)$ :

«Les poètes ont nommé le pays . . . les romanciers ont exploré le territoire de notre imaginaire. Les essayistes ont défini les fondements de nos libertés!"

Voilà. J'ai essayé de sonder l'essai. Articulation d'un articulet. Mais à quoi l'exigence du retour ou du repli correspond-elle ? Roland Barthes avait une réponse de son point de vue : "Rien n'est plus essentiel à une société que le classement de ses langages ". Mais comment les classer sans, d'abord, les analyser ?

Département de philosophie Université du Québec à Trois-Rivières 